\title{
Some results on degenerate Daehee and Bernoulli numbers and polynomials
}

\author{
Taekyun Kim,2, Dae San Kim ${ }^{4}$, Han Young Kim² and Jongkyum Kwon ${ }^{3 *}$
}

\section{"Correspondence:}

mathkjk26@gnu.ac.kr

${ }^{3}$ Department of Mathematics

Education and ERI, Gyeongsang

National University, Jinju, Republic

of Korea

Full list of author information is

available at the end of the article

\begin{abstract}
In this paper, we study a degenerate version of the Daehee polynomials and numbers, namely the degenerate Daehee polynomials and numbers, which were actually called the degenerate Daehee polynomials and numbers of the third kind and recently introduced by Jang et al. (J. Comput. Appl. Math. 364:112343, 2020). We derive their explicit expressions and some identities involving them. Further, we introduce the multiple degenerate Daehee numbers and higher-order degenerate Daehee polynomials and numbers which can be represented in terms of integrals on the unitcube. Again, we deduce their explicit expressions and some identities related to them.
\end{abstract}

MSC: $11 \mathrm{~B} 83 ; 11 \mathrm{~B} 73 ; 05 \mathrm{~A} 19$

Keywords: Degenerate Daehee polynomials and numbers; Multiple degenerate Daehee numbers; Higher-order degenerate Daehee polynomials and numbers

\section{Introduction}

The degenerate versions of Bernoulli and Euler polynomials, namely the degenerate Bernoulli and Euler polynomials, were studied by Carlitz in [1]. In recent years, studying various degenerate versions of some special polynomials and numbers drew attention of some mathematicians and many arithmetic and combinatorial results were obtained $[4,5,8,12,13,15-17,19,20]$. They can be explored by using various tools like combinatorial methods, generating functions, differential equations, umbral calculus techniques, $p$-adic analysis, and probability theory.

The aim of this paper is to study a degenerate version of the Daehee polynomials and numbers, namely the degenerate Daehee polynomials and numbers, in the spirit of [1]. They were actually called the degenerate Daehee polynomials and numbers of the third kind and recently introduced by Jang et al. in [4]. We derive their explicit expressions and some identities involving them. Further, we introduce the multiple degenerate Daehee numbers and higher-order degenerate Daehee polynomials and numbers. Again, we deduce their explicit expressions and some identities related to them.

This paper is organized as follows. In Sect. 1, we state what we need in the rest of the paper. These include the Stirling numbers of the first and second kinds, the higher-order Bernoulli polynomials, the higher-order Daehee polynomials, the higher-order degener-

(c) The Author(s) 2020. This article is licensed under a Creative Commons Attribution 4.0 International License, which permits use sharing, adaptation, distribution and reproduction in any medium or format, as long as you give appropriate credit to the original author(s) and the source, provide a link to the Creative Commons licence, and indicate if changes were made. The images or other third party material in this article are included in the article's Creative Commons licence, unless indicated otherwise in a credit line to the material. If material is not included in the article's Creative Commons licence and your intended use is not permitted by statutory regulation or exceeds the permitted use, you will need to obtain permission directly from the copyright holder. To view a copy of this licence, visit http://creativecommons.org/licenses/by/4.0/. 
ate Bernoulli polynomials, the degenerate exponential functions, and the degenerate Stirling numbers of the first and second kinds. In Sect. 2, we recall the degenerate Daehee polynomials and numbers (of the third kind) from [4] whose generating functions can be expressed in terms of integrals on the unit interval. We find their explicit expressions and some identities involving them. We also introduce the multiple degenerate Daehee numbers, the generating function of which can be expressed in terms of a multiple integral on the unitcube or of the modified polyexponential function [7]. We deduce an explicit expression of them and some identities involving them. In Sect. 3, we introduce the higherorder degenerate Daehee polynomials and numbers whose generating function can be represented as a multiple integral on the unitcube. We derive their explicit expressions and some identities relating to them. Finally, we conclude this paper in Sect. 4.

For $n \geq 0$, the Stirling numbers of the first kind are defined by

$$
(x)_{n}=\sum_{l=0}^{n} S_{1}(n, l) x^{l} \quad(\text { see }[8,14])
$$

where $(x)_{0}=1,(x)_{n}=x(x-1) \cdots(x-n+1),(n \geq 1)$.

As an inversion formula of (1.1), the Stirling numbers of the second kind are defined as

$$
x^{n}=\sum_{l=0}^{n} S_{2}(n, l)(x)_{l},(n \geq 0) \quad(\text { see }[8,14,16])
$$

For $\alpha \in \mathbb{N}$, the Bernoulli polynomials of order $\alpha$ are defined as

$$
\left(\frac{t}{e^{t}-1}\right)^{\alpha} e^{x t}=\sum_{n=0}^{\infty} B_{n}^{(\alpha)}(x) \frac{t^{n}}{n !} \quad(\text { see }[4,16])
$$

$B_{n}(x)=B_{n}^{(1)}(x)$ are called the Bernoulli polynomials and $B_{n}^{(\alpha)}=B_{n}^{(\alpha)}(0)$ the Bernoulli numbers of order $\alpha$.

The Daehee polynomials are defined by

$$
\left(\frac{\log (1+t)}{t}\right)(1+t)^{x}=\sum_{n=0}^{\infty} D_{n}(x) \frac{t^{n}}{n !} \quad(\text { see }[2,3,6,9-11,14,15,18,21-34]) .
$$

For $x=0, D_{n}=D_{n}(0)$ are called the Daehee numbers.

Recently, Jang-Kim-Kwon-Kim studied some new results on degenerate Daehee polynomials and numbers of the third kind (see [4]).

That is, they derived a new integral representation for the degenerate Daehee number and polynomials, the higher-order $\lambda$-Daehee numbers and polynomials, and the higherorder twisted $\lambda$-Daehee numbers and polynomials (see [4]).

The Daehee polynomials of order $k$ are defined by

$$
\left(\frac{\log (1+t)}{t}\right)^{k}(1+t)^{x}=\sum_{n=0}^{\infty} D_{n}^{(k)}(x) \frac{t^{n}}{n !} \quad(\text { see }[4,10])
$$


In [9], we note that

$$
D_{m}^{(k)}(z)=m ! \sum_{n=0}^{m}\left(\begin{array}{c}
z \\
m-n
\end{array}\right) b_{m}^{(-k)}
$$

where $b_{n}^{(x)}$ are the higher-order Bernoulli numbers of the second kind given by

$$
\left(\frac{t}{\log (1+t)}\right)^{x}=\sum_{n=0}^{\infty} b_{n}^{(x)} t^{n}
$$

Recently, Daehee numbers and polynomials have been studied by many researchers in various areas (see [2, 3, 6, 9-11, 14, 15, 18, 21-34]).

In [1], Carlitz considered the degenerate Bernoulli polynomials given by

$$
\frac{t}{(1+\lambda t)^{\frac{1}{\lambda}}-1}(1+\lambda t)^{\frac{x}{\lambda}}=\sum_{n=0}^{\infty} \beta_{n, \lambda}^{(x)} \frac{t^{n}}{n !} \quad(\lambda \in \mathbb{R})
$$

When $x=0, \beta_{n, \lambda}=\beta_{n, \lambda}(0)$ are called the degenerate Bernoulli numbers. For $r \in \mathbb{N}$, he also defined the higher-order degenerate Bernoulli polynomials as

$$
\left(\frac{t}{(1+\lambda t)^{\frac{1}{\lambda}}-1}\right)^{r}(1+\lambda t)^{\frac{x}{\lambda}}=\sum_{n=0}^{\infty} \beta_{n, \lambda}^{(r)}(x) \frac{t^{n}}{n !} \quad(\text { see }[16])
$$

When $x=0, \beta_{n, \lambda}^{(r)}=\beta_{n, \lambda}^{(r)}(0)$ are called the degenerate Bernoulli numbers of order $r$.

The degenerate exponential functions are given by

$$
e_{\lambda}^{x}(t)=(1+\lambda t)^{\frac{x}{\lambda}}, e_{\lambda}(t)=e_{\lambda}^{1}(t)=(1+\lambda t)^{\frac{1}{\lambda}} \quad(\text { see }[7-9,12-14,16-20])
$$

We note that

$$
e_{\lambda}^{x}(t)=\sum_{n=0}^{\infty} \frac{(x)_{n, \lambda}}{n !} t^{n} \quad(\text { see }[8])
$$

where $(x)_{0, \lambda}=1,(x)_{n, \lambda}=x(x-\lambda) \cdots(x-(n-1) \lambda)(n \geq 1)$.

Note that $\lim _{\lambda \rightarrow 0} e_{\lambda}^{x}(t)=e^{x t}, \lim _{\lambda \rightarrow 0} \beta_{n, \lambda}^{(r)}(x)=B_{n}^{(r)}(x)$.

Recently, Kim considered the degenerate Stirling numbers of the second kind given by

$$
(x)_{n, \lambda}=\sum_{l=0}^{n} S_{2, \lambda}(n, l)(x)_{l},(n \geq 0) \quad(\text { see }[8]) .
$$

Note that $\lim _{\lambda \rightarrow 0} S_{2, \lambda}(n, l)=S_{2}(n, l)$.

From (1.12), we note that

$$
\frac{1}{k !}\left(e_{\lambda}(t)-1\right)^{k}=\sum_{n=k}^{\infty} S_{2, \lambda}(n, k) \frac{t^{n}}{n !} \quad(k \geq 0)(\text { see }[8]) .
$$


As an inversion formula of (1.12), the Stirling numbers of the first kind are defined by

$$
(x)_{n}=\sum_{l=0}^{n} S_{1, \lambda}(n, l)(x)_{l, \lambda} \quad(n \geq 0)(\text { see }[8]) .
$$

We see that $\log _{\lambda}(t)=\frac{1}{\lambda}\left(t^{\lambda}-1\right)$ is the compositional inverse of $e_{\lambda}(t)$ satisfying $\log _{\lambda}\left(e_{\lambda}(t)\right)=$ $e_{\lambda}\left(\log _{\lambda}(t)\right)=t$.

By (1.14), we get

$$
\frac{1}{k !}\left(\log _{\lambda}(1+t)\right)^{k}=\sum_{n=k}^{\infty} S_{1, \lambda}(n, k) \frac{t^{n}}{n !} \quad(\text { see }[8]) .
$$

Note that $\lim _{\lambda \rightarrow 0} \log _{\lambda}(1+t)=\log (1+t)$.

\section{Degenerate Daehee numbers and polynomials}

The degenerate Daehee polynomials are defined by (see [4])

$$
\frac{\log _{\lambda}(1+t)}{t}(1+t)^{x}=\sum_{n=0}^{\infty} D_{n, \lambda}(x) \frac{t^{n}}{n !}, \quad(\lambda \in \mathbb{R}) .
$$

When $x=0, D_{n, \lambda}=D_{n, \lambda}(0)$ are called the degenerate Daehee numbers.

From (1.4) and (2.1), we note that $\lim _{\lambda \rightarrow 0} D_{n, \lambda}(x)=D_{n}(x)(n \geq 0)$.

We observe that

$$
\frac{\log (1+t)}{t} \int_{0}^{1}(1+t)^{\lambda y+x} d y=\frac{\log _{\lambda}(1+t)}{t}(1+t)^{x}=\sum_{n=0}^{\infty} D_{n, \lambda}(x) \frac{t^{n}}{n !}
$$

When $x=0$, we have

$$
\frac{\log (1+t)}{t} \int_{0}^{1}(1+t)^{\lambda y} d y=\sum_{n=0}^{\infty} D_{n, \lambda} \frac{t^{n}}{n !}
$$

On the other hand,

$$
\begin{aligned}
& \frac{\log (1+t)}{t} \int_{0}^{1}(1+t)^{\lambda y} d y \\
& =\frac{\log (1+t)}{t} \sum_{m=0}^{\infty} \frac{\lambda^{m}(\log (1+t))^{m}}{(m+1) !} \\
& =\frac{1}{t} \sum_{m=0}^{\infty} \frac{(\log (1+t))^{m+1}}{(m+1) !} \lambda^{m}=\frac{1}{t} \sum_{m=1}^{\infty} \lambda^{m-1} \frac{1}{m !}(\log (1+t))^{m} \\
& =\frac{1}{t} \sum_{m=1}^{\infty} \lambda^{m-1} \sum_{n=m}^{\infty} S_{1}(n, m) \frac{t^{n}}{n !}=\frac{1}{t} \sum_{n=1}^{\infty}\left(\sum_{m=1}^{n} \lambda^{m-1} S_{1}(n, m)\right) \frac{t^{n}}{n !} \\
& =\sum_{n=0}^{\infty}\left(\frac{1}{n+1} \sum_{m=1}^{n+1} \lambda^{m-1} S_{1}(n+1, m)\right) \frac{t^{n}}{n !}
\end{aligned}
$$

Therefore, by (2.2) and (2.3), we obtain the following theorem. 
Theorem 2.1 For $n \geq 0$, we have

$$
D_{n, \lambda}=\frac{1}{n+1} \sum_{m=1}^{n+1} \lambda^{m-1} S_{1}(n+1, m) .
$$

By replacing $t$ by $e_{\lambda}(t)-1$ in (2.1), we get

$$
\begin{aligned}
\frac{t}{e_{\lambda}(t)-1} e_{\lambda}^{x}(t) & =\sum_{m=0}^{\infty} D_{m, \lambda}(x) \frac{1}{m !}\left(e_{\lambda}(t)-1\right)^{m} \\
& =\sum_{m=0}^{\infty} D_{m, \lambda}(x) \sum_{n=m}^{\infty} S_{2, \lambda}(n, m) \frac{t^{n}}{n !} \\
& =\sum_{n=0}^{\infty}\left(\sum_{m=0}^{n} D_{m, \lambda}(x) S_{2, \lambda}(n, m)\right) \frac{t^{n}}{n !} .
\end{aligned}
$$

On the other hand,

$$
\frac{t}{e_{\lambda}(t)-1} e_{\lambda}^{x}(t)=\sum_{n=0}^{\infty} \beta_{n, \lambda}(x) \frac{t^{n}}{n}
$$

Therefore, by (2.4) and (2.5), we obtain the following theorem.

Theorem 2.2 For $n \geq 0$, we have

$$
\beta_{n, \lambda}(x)=\sum_{m=0}^{n} D_{m, \lambda}(x) S_{2, \lambda}(n, m) .
$$

Note that

$$
B_{n}(x)=\lim _{\lambda \rightarrow 0} \beta_{n, \lambda}(x)=\sum_{m=0}^{n} D_{m}(x) S_{2}(n, m) \quad(n \geq 0) .
$$

To find the inversion formula of Theorem 2.2, we replace $t$ by $\log _{\lambda}(1+t)$ in $(1.8)$ and get

$$
\begin{aligned}
\frac{\log _{\lambda}(1+t)}{t}(1+t)^{x} & =\sum_{m=0}^{\infty} \beta_{m, \lambda}(x) \frac{1}{m !}\left(\log _{\lambda}(1+t)\right)^{m} \\
& =\sum_{m=0}^{\infty} \beta_{m, \lambda}(x) \sum_{n=m}^{\infty} S_{1, \lambda}(n, m) \frac{t^{n}}{n !} \\
& =\sum_{n=0}^{\infty}\left(\sum_{m=0}^{n} \beta_{m, \lambda}(x) S_{1, \lambda}(n, m)\right) \frac{t^{n}}{n !} .
\end{aligned}
$$

Therefore, by (2.1) and (2.6), we obtain the following theorem.

Theorem 2.3 For $n \geq 0$, we have

$$
D_{n, \lambda}(x)=\sum_{m=0}^{n} \beta_{m, \lambda}(x) S_{1, \lambda}(n, m) .
$$


Note that

$$
D_{n}(x)=\lim _{\lambda \rightarrow 0} D_{n, \lambda}(x)=\sum_{m=0}^{n} B_{m}(x) S_{1}(n, m) \quad(n \geq 0) .
$$

From (1.10), we can derive the following equation:

$$
\begin{aligned}
\sum_{n=0}^{\infty} D_{n, \lambda}(x) \frac{t^{n}}{n !} & =\frac{\log _{\lambda}(1+t)}{t}(1+t)^{x}=\frac{\log _{\lambda}(1+t)}{t} e_{\lambda}^{x}\left(\log _{\lambda}(1+t)\right) \\
& =\frac{\log _{\lambda}(1+t)}{t} \sum_{m=0}^{\infty}(x)_{m, \lambda} \frac{\left(\log _{\lambda}(1+t)\right)^{m}}{m !} \\
& =\frac{1}{t} \sum_{m=0}^{\infty}(m+1)(x)_{m, \lambda} \frac{1}{(m+1) !}\left(\log _{\lambda}(1+t)\right)^{m+1} \\
& =\frac{1}{t} \sum_{m=0}^{\infty}(m+1)(x)_{m, \lambda} \sum_{n=m+1}^{\infty} S_{1, \lambda}(n, m+1) \frac{t^{n}}{n !} \\
& =\sum_{m=0}^{\infty}(m+1)(x)_{m, \lambda} \sum_{n=m}^{\infty} \frac{S_{1, \lambda}(n+1, m+1)}{n+1} \frac{t^{n}}{n !} \\
& =\sum_{n=0}^{\infty}\left\{\frac{1}{n+1} \sum_{m=0}^{n}(m+1)(x)_{m, \lambda} S_{1, \lambda}(n+1, m+1)\right\} \frac{t^{n}}{n !} .
\end{aligned}
$$

Therefore, by (2.7), we obtain the following theorem.

Theorem 2.4 For $n \geq 0$, we have

$$
D_{n, \lambda}(x)=\frac{1}{n+1} \sum_{m=0}^{n}(m+1)(x)_{m, \lambda} S_{1, \lambda}(n+1, m+1) .
$$

For $s \in \mathbb{C}$, the polyexponential function is defined by Hardy as

$$
e(x, a \mid s)=\sum_{n=0}^{\infty} \frac{x^{n}}{(n+a)^{s} n !},(\operatorname{Re}(a)>0) \quad(\text { see }[16]) .
$$

In [7], the modified polyexponential function is introduced as

$$
\mathrm{Ei}_{k}(x)=\sum_{n=1}^{\infty} \frac{x^{n}}{(n-1) ! n^{k}} \quad(k \in \mathbb{Z}) .
$$

Note that $x e(x, 1 \mid k)=\operatorname{Ei}_{k}(x)$.

We observe that

$$
\frac{\partial}{\partial x_{1}}(1+t)^{\lambda x_{1} x_{2} \cdots x_{k}}=x_{2} \cdots x_{k} \lambda \log (1+t)(1+t)^{\lambda x_{1} x_{2} \cdots x_{k}}
$$


Thus, by (2.11), we get

$$
\begin{aligned}
& \frac{\log (1+t)}{t} \int_{0}^{1}(1+t)^{\lambda x_{1} x_{2} \cdots x_{k}} d x_{1} \\
& =\frac{1}{x_{2} x_{3} \cdots x_{k}} \frac{\log _{\lambda}(1+t)^{x_{2} \cdots x_{k}}}{t} \\
& =\frac{1}{t} \frac{1}{x_{2} x_{3} \cdots x_{k}} \sum_{m=1}^{\infty} \lambda^{m-1} \frac{(\log (1+t))^{m}}{m !} x_{2}^{m} x_{3}^{m} \cdots x_{k}^{m} \\
& =\frac{1}{t} \sum_{m=1}^{\infty} \frac{\lambda^{m-1}(\log (1+t))^{m}}{(m-1) ! m} x_{2}^{m-1} x_{3}^{m-1} \cdots x_{k}^{m-1} .
\end{aligned}
$$

From (2.12), we can derive the following equation:

$$
\begin{gathered}
\frac{\log (1+t)}{t} \int_{0}^{1} \cdots \int_{0}^{1}(1+t)^{\lambda x_{1} x_{2} \cdots x_{k}} d x_{1} d x_{2} \cdots d x_{k} \\
=\frac{1}{t} \sum_{m=1}^{\infty} \frac{\lambda^{m-1}(\log (1+t))^{m}}{(m-1) ! m^{k}}=\frac{1}{\lambda t} \operatorname{Ei}_{k}(\lambda \log (1+t)) .
\end{gathered}
$$

Now, we define the multiple degenerate Daehee numbers as the multiple integral on the unitcube given by

$$
\frac{\log (1+t)}{t} \int_{0}^{1} \cdots \int_{0}^{1}(1+t)^{\lambda x_{1} x_{2} \cdots x_{k}} d x_{1} d x_{2} \cdots d x_{k}=\sum_{n=0}^{\infty} \widehat{D}_{n, \lambda}^{(k)} \frac{t^{n}}{n !} .
$$

Then, by (2.13) and (2.14), we get

$$
\frac{1}{\lambda t} \operatorname{Ei}_{k}(\lambda \log (1+t))=\sum_{n=0}^{\infty} \widehat{D}_{n, \lambda}^{(k)} \frac{t^{n}}{n !}
$$

Note that $\widehat{D}_{n, \lambda}^{(1)}=D_{n, \lambda}(n \geq 0)$.

We observe that

$$
\begin{aligned}
\frac{1}{\lambda t} \operatorname{Ei}_{k}(\lambda \log (1+t)) & =\frac{1}{\lambda t} \sum_{m=1}^{\infty} \frac{\lambda^{m}(\log (1+t))^{m}}{(m-1) ! m^{k}} \\
& =\frac{1}{\lambda t} \sum_{m=1}^{\infty} \frac{\lambda^{m}}{m^{k-1}} \frac{1}{m !}(\log (1+t))^{m} \\
& =\frac{1}{\lambda t} \sum_{m=1}^{\infty} \frac{\lambda^{m}}{m^{k-1}} \sum_{n=m}^{\infty} S_{1}(n, m) \frac{t^{n}}{n !} \\
& =\frac{1}{t} \sum_{n=1}^{\infty} \sum_{m=1}^{n} \frac{\lambda^{m-1}}{m^{k-1}} S_{1}(n, m) \frac{t^{n}}{n !} \\
& =\sum_{n=0}^{\infty}\left(\frac{1}{n+1} \sum_{m=1}^{n+1} \frac{\lambda^{m-1}}{m^{k-1}} S_{1}(n+1, m)\right) \frac{t^{n}}{n !} .
\end{aligned}
$$

Therefore, by (2.15) and (2.16), we obtain the following theorem. 
Theorem 2.5 For $n \geq 0$, we have

$$
\widehat{D}_{n, \lambda}^{(k)}=\frac{1}{n+1} \sum_{m=1}^{n+1} \frac{\lambda^{m-1}}{m^{k-1}} S_{1}(n+1, m) .
$$

By replacing $t$ by $e^{t}-1$ in (2.15), we get

$$
\begin{aligned}
\sum_{m=0}^{\infty} \widehat{D}_{m, \lambda}^{(k)} \frac{1}{m !}\left(e^{t}-1\right)^{m} & =\frac{1}{\lambda\left(e^{t}-1\right)} \operatorname{Ei}_{k}(\lambda t) \\
& =\frac{t}{e^{t}-1} \frac{1}{\lambda t} \operatorname{Ei}_{k}(\lambda t)=\sum_{l=0}^{\infty} B_{l} \frac{t^{l}}{l !} \sum_{m=0}^{\infty} \frac{\lambda^{m}}{(m+1)^{k}} \frac{t^{m}}{m !} \\
& =\sum_{n=0}^{\infty}\left(\sum_{l=0}^{n}\left(\begin{array}{l}
n \\
l
\end{array}\right) \frac{\lambda^{n-l} B_{l}}{(n-l+1)^{k}}\right) \frac{t^{n}}{n !} .
\end{aligned}
$$

On the other hand,

$$
\begin{aligned}
\sum_{m=0}^{\infty} \widehat{D}_{m, \lambda}^{(k)} \frac{1}{m !}\left(e^{t}-1\right)^{m} & =\sum_{m=0}^{\infty} \widehat{D}_{m, \lambda}^{(k)} \sum_{n=m}^{\infty} S_{2}(n, m) \frac{t^{n}}{n !} \\
& =\sum_{n=0}^{\infty}\left(\sum_{m=0}^{n} \widehat{D}_{m, \lambda}^{(k)} S_{2}(n, m)\right) \frac{t^{n}}{n !}
\end{aligned}
$$

Therefore, by (2.17) and (2.18), we obtain the following theorem.

Theorem 2.6 For $n \geq 0$, we have

$$
\sum_{m=0}^{n} \widehat{D}_{m, \lambda}^{(k)} S_{2}(n, m)=\sum_{l=0}^{n}\left(\begin{array}{l}
n \\
l
\end{array}\right) \frac{\lambda^{n-l} B_{l}}{(n-l+1)^{k}} .
$$

From (2.17), we note that

$$
\begin{aligned}
\frac{1}{\lambda t} \mathrm{Ei}_{k}(\lambda t) & =\frac{1}{t}\left(e^{t}-1\right) \sum_{m=0}^{\infty} \widehat{D}_{m, \lambda}^{(k)} \frac{1}{m !}\left(e^{t}-1\right)^{m} \\
& =\frac{1}{t} \sum_{m=1}^{\infty} m \widehat{D}_{m-1, \lambda}^{(k)} \frac{1}{m !}\left(e^{t}-1\right)^{m} \\
& =\frac{1}{t} \sum_{m=1}^{\infty} m \widehat{D}_{m-1, \lambda}^{(k)} \sum_{n=m}^{\infty} S_{2}(n, m) \frac{t^{n}}{n !} \\
& =\frac{1}{t} \sum_{n=1}^{\infty} \sum_{m=1}^{n} m \widehat{D}_{m-1, \lambda}^{(k)} S_{2}(n, m) \frac{t^{n}}{n !} \\
& =\sum_{n=0}^{\infty}\left(\frac{1}{n+1} \sum_{m=1}^{n+1} m \widehat{D}_{m-1, \lambda}^{(k)} S_{2}(n+1, m)\right) \frac{t^{n}}{n !}
\end{aligned}
$$


On the other hand,

$$
\frac{1}{\lambda t} \mathrm{Ei}_{k}(\lambda t)=\frac{1}{\lambda t} \sum_{n=1}^{\infty} \frac{\lambda^{n} t^{n}}{(n-1) ! n^{k}}=\sum_{n=0}^{\infty} \frac{\lambda^{n}}{(n+1)^{k}} \frac{t^{n}}{n !}
$$

Therefore, by (2.19) and (2.20), we obtain the following theorem.

Theorem 2.7 For $n \geq 0$, we have

$$
\begin{aligned}
\frac{\lambda^{n}}{(n+1)^{k}} & =\frac{1}{n+1} \sum_{m=1}^{n} m \widehat{D}_{m-1, \lambda}^{(k)} S_{2}(n+1, m) \\
& =\frac{1}{n+1} \sum_{m=0}^{n-1}(m+1) \widehat{D}_{m, \lambda}^{(k)} S_{2}(n+1, m+1) .
\end{aligned}
$$

\section{Higher-order degenerate Daehee numbers and polynomials}

As an additive version of (2.14), we consider the degenerate Daehee polynomials of order $r$ given by the following multiple integral on the unit cube:

$$
\begin{aligned}
\sum_{n=0}^{\infty} D_{n, \lambda}^{(r)}(x) \frac{t^{n}}{n !} & =\left(\frac{\log (1+t)}{t}\right)^{r} \int_{0}^{1} \cdots \int_{0}^{1}(1+t)^{\lambda\left(x_{1}+\cdots+x_{r}\right)+x} d x_{1} \cdots d x_{r} \\
& =\left(\frac{\log _{\lambda}(1+t)}{t}\right)^{r}(1+t)^{x} \quad(r \in \mathbb{N}) .
\end{aligned}
$$

When $x=0, D_{n, \lambda}^{(r)}=D_{n, \lambda}^{(r)}(0)(n \geq 0)$, are called the degenerate Daehee numbers of order $r$.

From (3.1), we note that

$$
\begin{aligned}
\sum_{n=0}^{\infty} D_{n, \lambda}^{(r)} \frac{t^{n}}{n !} & =\left(\frac{\log _{\lambda}(1+t)}{t}\right)^{r}=\frac{r !}{t^{r}} \frac{1}{r !}\left(\log _{\lambda}(1+t)\right)^{r} \\
& =\frac{r !}{t^{r}} \sum_{n=r}^{\infty} S_{1, \lambda}(n, r) \frac{t^{n}}{n !} \\
& =\sum_{n=0}^{\infty} S_{1, \lambda}(n+r, r) \frac{r ! n !}{(n+r) !} \frac{t^{n}}{n !} \\
& =\sum_{n=0}^{\infty} \frac{S_{1, \lambda}(n+r, r)}{\left(\begin{array}{c}
n+r \\
n
\end{array}\right)} \frac{t^{n}}{n !} .
\end{aligned}
$$

Therefore, by comparing the coefficients on both sides of (3.2), we obtain the following theorem.

Theorem 3.1 For $n \geq 0$, we have

$$
D_{n, \lambda}^{(r)}=\frac{1}{\left(\begin{array}{c}
n+r \\
n
\end{array}\right)} S_{1, \lambda}(n+r, r) \quad(r \in \mathbb{N}) .
$$


By replacing $t$ by $e_{\lambda}(t)-1$ in (3.1), we get

$$
\begin{aligned}
\sum_{k=0}^{\infty} D_{k, \lambda}^{(r)}(x) \frac{1}{k !}\left(e_{\lambda}(t)-1\right)^{k} & =\left(\frac{t}{e_{\lambda}(t)-1}\right)^{r} e_{\lambda}^{x}(t) \\
& =\sum_{n=0}^{\infty} \beta_{n, \lambda}^{(r)}(x) \frac{t^{n}}{n !}
\end{aligned}
$$

On the other hand,

$$
\begin{aligned}
& \sum_{k=0}^{\infty} D_{k, \lambda}^{(r)}(x) \frac{1}{k !}\left(e_{\lambda}(t)-1\right)^{k} \\
& =\sum_{k=0}^{\infty} D_{k, \lambda}^{(r)}(x) \sum_{n=k}^{\infty} S_{2, \lambda}(n, k) \frac{t^{n}}{n !} \\
& =\sum_{n=0}^{\infty}\left(\sum_{k=0}^{\infty} D_{k, \lambda}^{(r)}(x) S_{2, \lambda}(n, k)\right) \frac{t^{n}}{n !} .
\end{aligned}
$$

Therefore, by (3.3) and (3.4), we obtain the following theorem.

Theorem 3.2 For $n \geq 0$, we have

$$
\beta_{n, \lambda}^{(r)}(x)=\sum_{k=0}^{n} D_{k, \lambda}^{(r)}(x) S_{2, \lambda}(n, k) .
$$

By replacing $t$ by $\log _{\lambda}(1+t)$ in (1.9), we get

$$
\begin{aligned}
\left(\frac{\log _{\lambda}(1+t)}{t}\right)^{r}(1+t)^{x} & =\sum_{k=0}^{\infty} \beta_{k, \lambda}^{(r)}(x) \frac{1}{k !}\left(\log _{\lambda}(1+t)\right)^{k} \\
& =\sum_{k=0}^{\infty} \beta_{k, \lambda}^{(r)}(x) \sum_{n=k}^{\infty} S_{1, \lambda}(n, k) \frac{t^{n}}{n !} \\
& =\sum_{n=0}^{\infty}\left(\sum_{k=0}^{n} \beta_{k, \lambda}^{(r)}(x) S_{1, \lambda}(n, k)\right) \frac{t^{n}}{n !}
\end{aligned}
$$

On the other hand,

$$
\left(\frac{\log _{\lambda}(1+t)}{t}\right)^{r}(1+t)^{x}=\sum_{n=0}^{\infty} D_{n, \lambda}^{(r)}(x) \frac{t^{n}}{n !}
$$

Therefore, by (3.5) and (3.6), we obtain the following theorem.

Theorem 3.3 For $n \geq 0$, we have

$$
D_{n, \lambda}^{(r)}(x)=\sum_{k=0}^{n} \beta_{k, \lambda}^{(r)}(x) S_{1, \lambda}(n, k) .
$$


From (3.1), we note that

$$
\begin{aligned}
\sum_{n=0}^{\infty} D_{n, \lambda}^{(r)} \frac{t^{n}}{n !} & =\underbrace{\left(\frac{\log _{\lambda}(1+t)}{t}\right) \times \cdots \times \frac{\log _{\lambda}(1+t)}{t}}_{r \text {-times }} \\
& =\sum_{n=0}^{\infty}\left(\sum_{l_{1}+\cdots+l_{r}=n}\left(\begin{array}{c}
n \\
l_{1}, \ldots l_{r}
\end{array}\right) D_{l_{1}, \lambda} \cdots D_{l_{r}, \lambda}\right) \frac{t^{n}}{n !} .
\end{aligned}
$$

By (3.7), we get

$$
D_{n, \lambda}^{(r)}=\sum_{l_{1}+\cdots+l_{r}=n}\left(\begin{array}{c}
n \\
l_{1}, \ldots l_{r}
\end{array}\right) D_{l_{1}, \lambda} \cdots D_{l_{r}, \lambda} \quad(n \geq 0) .
$$

On the other hand, by (3.2), we get

$$
\begin{aligned}
& \sum_{n=0}^{\infty} D_{n, \lambda}^{(r)} \frac{t^{n}}{n !}=\left(\frac{\log (1+t)}{t}\right)^{r} \int_{0}^{1} \cdots \int_{0}^{1}(1+t)^{\lambda\left(x_{1}+\cdots+x_{r}\right)} d x_{1} \cdots d x_{r} \\
& =\left(\frac{\log (1+t)}{t}\right)^{r} \sum_{m=0}^{\infty} \lambda^{m} \frac{(\log (1+t))^{m}}{m !} \int_{0}^{1} \cdots \int_{0}^{1}\left(x_{1}+\cdots+x_{r}\right)^{m} d x_{1} \cdots d x_{r} \\
& =\frac{1}{t^{r}} \sum_{m=0}^{\infty} \lambda^{m} \sum_{l_{1}+\cdots+l_{r}=m}\left(\begin{array}{c}
m \\
l_{1}, \ldots, l_{r}
\end{array}\right) \frac{1}{\left(l_{1}+1\right) \cdots\left(l_{r}+1\right)} \frac{(\log (1+t))^{m+r}}{m !} \\
& =\frac{1}{t^{r}} \sum_{m=0}^{\infty} \lambda^{m} \sum_{l_{1}+\cdots+l_{r}=m}\left(\begin{array}{c}
m \\
l_{1}, \ldots, l_{r}
\end{array}\right) \frac{1}{\left(l_{1}+1\right) \cdots\left(l_{r}+1\right)} \frac{(m+r) !}{m !} \\
& \times \sum_{n=m+r}^{\infty} S_{1}(n, m+r) \frac{t^{n}}{n !} \\
& =\sum_{m=0}^{\infty} \lambda^{m} \sum_{l_{1}+\cdots+l_{r}=m}\left(\begin{array}{c}
m \\
l_{1}, \ldots, l_{r}
\end{array}\right) \frac{1}{\left(l_{1}+1\right) \cdots\left(l_{r}+1\right)} \frac{(m+r) !}{m !} \\
& \times \sum_{n=m}^{\infty} S_{1}(n+r, m+r) \frac{t^{n}}{(n+r) !} \\
& =\sum_{n=0}^{\infty}\left(\sum_{m=0}^{n} \lambda^{m} \sum_{l_{1}+\cdots+l_{r}=m} \frac{\left(\begin{array}{c}
n \\
l_{1}, \ldots, l_{r}
\end{array}\right)}{\left(l_{1}+1\right) \cdots\left(1_{r}+1\right)} S_{1}(n+r, m+r) \frac{\left(\begin{array}{c}
m+r \\
r
\end{array}\right)}{\left(\begin{array}{c}
n+r \\
r
\end{array}\right)}\right) \frac{t^{n}}{n !} .
\end{aligned}
$$

Therefore, by comparing the coefficients on both sides of (3.9), we obtain the following theorem.

Theorem 3.4 For $n \geq 0$, we have

$$
\begin{aligned}
D_{n, \lambda}^{(r)} & =\sum_{l_{1}+\cdots+l_{r}=m}\left(\begin{array}{c}
n \\
l_{1}, \ldots, l_{r}
\end{array}\right) D_{l_{1}, \lambda} \cdots D_{l_{r}, \lambda} \\
& =\sum_{m=0}^{n} \lambda^{m} \sum_{l_{1}+\cdots+l_{r}=m}\left(\begin{array}{c}
m \\
l_{1}, \ldots, l_{r}
\end{array}\right) \frac{S_{1}(n+r, m+r)}{\left(l_{1}+1\right) \cdots\left(1_{r}+1\right)} \frac{\left(\begin{array}{c}
m+r \\
r
\end{array}\right)}{\left(\begin{array}{c}
n+r \\
r
\end{array}\right)} .
\end{aligned}
$$




\section{Conclusion}

In the spirit of [1], we studied the degenerate Daehee polynomials and numbers which were actually called the degenerate Daehee polynomials and numbers of the third kind and recently introduced by Jang et al. in [4]. We derived their explicit expressions and some identities involving them. Further, we introduced the multiple degenerate Daehee numbers and higher-order degenerate Daehee polynomials and numbers and deduced their explicit expressions and some identities related to them.

The possible applications of our results are as follows. The first one is their applications to identities of symmetry. For example, in [13] many symmetric identities in three variables, related to degenerate Euler polynomials and alternating generalized falling factorial sums, were obtained. The second one is their applications to differential equations from which we can derive some useful identities. For example, in [12] an infinite family of nonlinear differential equations, having the generating function of the degenerate Bernoulli numbers as a solution, were derived. As a result, an identity, involving the degenerate Bernoulli and higher-order degenerate Bernoulli numbers, were obtained. Similar things had been done for the degenerate Euler numbers. The third one is their applications to probability theory. Indeed, in [19] it was shown that both the degenerate $\lambda$-Stirling polynomials of the second and the $r$-truncated degenerate $\lambda$-Stirling polynomials of the second kind appear in certain expressions of the probability distributions of appropriate random variables.

Finally, it is one of our future projects to continue to study various degenerate versions of some special polynomials and their applications to mathematics, science and engineering.

We studied the degenerate Daehee polynomials and numbers which are different from the degenerate Daehee polynomials and numbers of the third kind introduced by Jang et al. [4].

\section{Acknowledgements}

We would like to thank the referees for their valuable comments and suggestions. Also, we would like to thank Jangjeon Institute for Mathematical Science for the support of this research.

Funding

This work was supported by the National Research Foundation of Korea (NRF) grant funded by the Korea government (MEST) (No. 2017R1E1A1A03070882).

Availability of data and materials

Not applicable.

Competing interests

The authors declare that they have no conflicts of interest.

Authors' contributions

TK and DSK conceived of the framework and structured the whole paper; DSK and TK wrote the paper; JK and HYK checked the results of the paper; DSK and TK completed the revision of the article. All authors have read and agreed to the published version of the manuscript.

\section{Author details}

${ }^{1}$ School of Science, Xian Technological University, Xian, China. ${ }^{2}$ Department of Mathematics, Kwangwoon University, Seoul, Republic of Korea. ${ }^{3}$ Department of Mathematics Education and ERI, Gyeongsang National University, Jinju,

Republic of Korea. ${ }^{4}$ Department of Mathematics, Sogang University, Seoul, Korea.

\section{Publisher's Note}

Springer Nature remains neutral with regard to jurisdictional claims in published maps and institutional affiliations. 


\section{References}

1. Carlitz, L.: Degenerate Stirling, Bernoulli and Eulerian numbers. Util. Math. 15, 51-88 (1979)

2. Dolgy, D.V., Jang, G.-W., Kim, D.S., Kim, T.: Explicit expressions for Catalan-Daehee numbers. Proc. Jangjeon Math. Soc. 20(1), 1-9 (2017)

3. El-Desouky, B.S., Mustafa, A.: New results on higher-order Daehee and Bernoulli numbers and polynomilas. Adv Differ. Equ. 2016, 206 (2016)

4. Jang, L.-C., Kim, W., Kwon, H.-I., Kim, T.: On degenerate Daehee polynomials and numbers of the third kind. J. Comput. Appl. Math. 364, Article ID 112343 (2020)

5. Jang, L.-C., Kim, D. S., Kim, T., Lee, H.: p-Adic integral on $\mathbb{Z}_{p}$ associated with degenerate Bernoulli polynomials of the second kind. Adv. Differ. Equ. 2020, Article ID 278 (2020)

6. Kim, D., Kim, T.: Idntities arising from higher-order Daehee polynomial bases. Open Math. 13(1), 196-208 (2015)

7. Kim, D.S., Kim, T.: A note on polyexponential and unipoly functions. Russ. J. Math. Phys. 26(1), 40-49 (2019)

8. Kim, D.S., Kim, T.: A note on a new type of degenerate Bernoulli numbers. Russ. J. Math. Phys. 27(2), 227-235 (2020)

9. Kim, D.S., Kim, T., Kwon, J., Lee, H.: A note on $\lambda$-Bernoulli numbers of the second kind. Adv. Stud. Contemp. Math. (Kyungshang) 30(2), 187-195 (2020)

10. Kim, D.S., Kim, T., Ryoo, C.S.: Generalized type 2 degenerate Euler numbers. Adv. Stud. Contemp. Math. (Kyungshang) 30(2), 165-169 (2020)

11. Kim, T., Jang, L.-C., Kim, D.S., Kim, H.Y.: Some identities on type 2 degenerate Bernoulli polynomials of the second kind Symmetry 12(4), Article ID 510 (2020)

12. Kim, T., Kim, D.S.: Identities involving degenerate Euler numbers and polynomials arising from non-linear differential equations. J. Nonlinear Sci. Appl. 9, 2086-2098 (2016)

13. Kim, T., Kim, D.S.: Identities of symmetry for degenerate Euler polynomials and alternating generalized falling factoria sums. Iran. J. Sci. Technol. Trans. A, Sci. 41(4), 939-949 (2017)

14. Kim, T., Kim, D.S.: A note on type 2 Changhee and Daehee polynomials. Rev. R. Acad. Cienc. Exactas Fís. Nat., Ser. A Mat. 113(3), 2783-2791 (2019)

15. Kim, T., Kim, D.S.: Extended Stirling numbers of the first kind associated with Daehee numbers and polynomials. Rev. R. Acad. Cienc. Exactas Fís. Nat., Ser. A Mat. 113(2), 1159-1171 (2019)

16. Kim, T., Kim, D.S.: Degenerate polyexponential functions and degenerate Bell polynomials. J. Math. Anal. Appl. 487(2), $124017(2020)$

17. Kim, T., Kim, D.S.: Degenerate binomial coefficients and degenerate hypergeometric functions. Adv. Differ. Equ. 2020, Article ID 115 (2020)

18. Kim, T., Kim, D.S.: A note on central Bell numbers and polynomials. Russ. J. Math. Phys. 27(1), 76-81 (2020)

19. Kim, T., Kim, D.S., Kim, H.Y., Kwon, J.: Degenerate Stirling polynomials of the second kind and some applications. Symmetry 11(8), $1046(2019)$

20. Kim, T., Kim, D.S., Kwon, J., Lee, H.: Degenerate polyexponential functions and type 2 degenerate poly-Bernoulli numbers and polynomials. Adv. Differ. Equ. 2020, 168 (2020)

21. Kwon, J., Kim, T., Kim, D.S., Kim, H.Y.: Some identities for degenerate complete and incomplete $r$-Bell polynomials. J. Inequal. Appl. 2020, Article ID 23 (2020)

22. Kwon, J., Kim, W.J., Rim, S.-H.: On the some identities of the type 2 Daehee and Changhee polynomials arising from p-adic integrals on $Z_{p}$. Proc. Jangjeon Math. Soc. 22(3), 487-497 (2019)

23. Liu, C., Wuyungaowa, B.: Application of probabilistic method on Daehee sequences. Eur. J. Pure Appl. Math. 11(1), 69-78 (2018)

24. Liu, G.-D., Srivastava, H.M.: Explicit formulas for the Nörlund polynomials $B_{n}^{(x)}$ and $b_{n}^{(x)}$. Comput. Math. Appl. 51, 1377-1384 (2006)

25. Luo, Y.-N., Wuyungaowa, B.: Some combinatorial identities about Daehee sequences. J. Comb. Math. Comb. Comput. 108, 75-87 (2019)

26. Moon, E.-J., Park, J.-W., Rim, S.-H.: A note on the generalized q-Daehee numbers of higher order. Proc. Jangjeon Math. Soc. 17(4), 557-565 (2014)

27. Park, J.-W.: On the $q$-analogue of Daehee numbers and polynomials. Proc. Jangjeon Math. Soc. 19(3), 537-544 (2016)

28. Park, J.-W., Rim, S.-H., Kwon, J.: The twisted Daehee numbers and polynomials. Adv. Differ. Equ. 2014, 1 (2014)

29. Pyo, S.-S., Kim, T., Rim, S.-S.: Degenerate Daehee numbers of the third kind. Mathematics 6(11), 239 (2018)

30. Roman, S.: The Umbral Calculus. Pure and Applied Mathematics, vol. 111. Acsdemic Press, New York (1984)

31. Saif, M., Nadeem, R.: Evaluation of Apostol-Euler based poly Daehee polynomials. Int. J. Appl. Comput. Math. 6, Article ID 1 (2020)

32. Seo, J.J., Rim, S.H., Kim, T., Lee, S.H.: Sums products of generalized Daehee numbers. Proc. Jangjeon Math. Soc. 17(1), 1-9 (2014)

33. Simsek, Y.: Apostol type Daehee numbers and polynomials. Adv. Stud. Contemp. Math. (Kyungshang) 26, 555-566 (2016)

34. Simsek, Y.: Identities on the Changhee numbers and Apostol-type Daehee polynomials. Adv. Stud. Contemp. Math. (Kyungshang) 27, 199-212 (2017) 